\title{
Interconnection of subsystems in closed-loop systems
}

\author{
Niemann, Hans Henrik; Poulsen, Niels Kjølstad
}

Published in:

Proceedings of the 48th IEEE Conference on Decision and Control, 2009 held jointly with the 2009 28th Chinese Control Conference. CDC/CCC 2009

Link to article, DOI:

10.1109/CDC.2009.5400817

Publication date:

2009

Document Version

Publisher's PDF, also known as Version of record

Link back to DTU Orbit

\section{Citation (APA):}

Niemann, H. H., \& Poulsen, N. K. (2009). Interconnection of subsystems in closed-loop systems. In Proceedings of the 48th IEEE Conference on Decision and Control, 2009 held jointly with the 2009 28th Chinese Control Conference. CDC/CCC 2009 (pp. 632-637). IEEE. https://doi.org/10.1109/CDC.2009.5400817

\section{General rights}

Copyright and moral rights for the publications made accessible in the public portal are retained by the authors and/or other copyright owners and it is a condition of accessing publications that users recognise and abide by the legal requirements associated with these rights.

- Users may download and print one copy of any publication from the public portal for the purpose of private study or research.

- You may not further distribute the material or use it for any profit-making activity or commercial gain

- You may freely distribute the URL identifying the publication in the public portal 


\section{Interconnection of Subsystems in Closed-loop Systems}

\author{
Henrik Niemann \\ Dept. of Elec. Engineering \\ Automation and Control \\ Tech. Univ. of Denmark, Build. 326 \\ DK-2800 Kgs. Lyngby, Denmark \\ hhn@elektro.dtu.dk
}

\author{
Niels Kjølstad Poulsen \\ Informatics and Math. Modelling \\ Tech. Univ. of Denmark, Build. 321 \\ DK-2800 Kgs. Lyngby, Denmark \\ nkp@imm.dtu.dk
}

\begin{abstract}
The focus in this paper is analysis of stability and controller design for interconnected systems. This includes both the case with known and unknown interconnected sub-system. The key element in both the stability analysis and controller design is the application of the Youla-Jabr-Bongiorno-Kucera (YJBK) parameterization. The dual YJBK transfer function is applied in connection with the closed-loop stability analysis. The primary YJBK parameterization is applied in connection with design of controllers. Further, it is shown how it is possible to obtain a direct estimation of a connected sub-system without having a direct access to it.
\end{abstract}

\section{INTRODUCTION}

The complexity of the control systems has been increasing the last years. The reason is the increasing requirements from the surrounding environments with respect to pollution, energy consumption, performance, cost etc. Examples are engines for cars, [4] and refrigeration systems. A number of these conditions can only be satisfied with the cost of more complex control systems.

The area of interconnected systems has been described and investigated in a large number of papers through the years. The modelling has been considered in details by J.C. Willems in a number of paper, see e.g. [8] and the reference herein. Design of controllers, specially distributed controllers has also been investigated, see e.g. [2], [10] and the references herein.

The first part of the paper deals with an analysis of closedloop systems when a sub-system is included in the closed loop. This includes a stability analysis in both the case when the sub-system is known and the case when it is unknown. When the sub-system is unknown, it is investigated how it is possible to get a direct estimation of the sub-system, when it is included in the closed-loop.

In the last part of the paper, design of feedback controllers for the interconnected system is considered. Two different controller concepts will be considered.

The rest of this paper is organized as follows. The applied system set-up is shortly described in Section II. Section III include a stability analysis of the closed-loop interconnected system. Controller design for interconnected systems are considered in Section IV followed by an example in Section $\mathrm{V}$. The paper is closed by a discussion in Section VI.

\section{SYSTEM SET-UP}

Let the main system $\Sigma_{1}$ and the subsystem $\Sigma_{2}$ be described by the following general system for $i=1,2$ :

$$
\Sigma_{i}:\left\{\begin{aligned}
e_{i} & =G_{e d, i} d_{i}+G_{e w, i} w_{i}+G_{e u, i} u_{i} \\
z_{i} & =G_{z d, i} d_{i}+G_{z w, i} w_{i}+G_{z u, i} u_{i} \\
y_{i} & =G_{y d, i} d_{i}+G_{y w, i} w_{i}+G_{y u, i} u_{i}
\end{aligned}\right.
$$

where $w_{i} \in \mathcal{R}^{q_{i}}$ is an external input, $d_{i} \in \mathcal{R}^{r_{i}}$ is a disturbance signal vector, $u_{i} \in \mathcal{R}^{m_{i}}$ the control input signal vector, $z_{i} \in$ $\mathcal{R}^{l_{i}}$ is an external output vector, $e_{i} \in \mathcal{R}^{k_{i}}$ is the external output signal vector to be controlled and $y_{i} \in \mathcal{R}^{p_{i}}$ is the measurement vector.

Further, let $\Sigma_{i}$ has the following state space representation:

$$
\Sigma_{i}:\left\{\begin{array}{l}
\dot{x}_{i}=A_{i} x+B_{d, i} d_{i}+B_{w, i} w_{i}+B_{u, i} u_{i} \\
e_{i}=C_{e, i} x_{i}+D_{e d, i} d_{i}+D_{e w, i} w_{i}+D_{e u, i} u_{i} \\
z_{i}=C_{z, i} x_{i}+D_{z d, i} d_{i}+D_{z w, i} w_{i}+D_{z u, i} u_{i} \\
y_{i}=C_{y, i} x_{i}+D_{y d, i} d_{i}+D_{y w, i} w_{i}+D_{y u, i} u_{i}
\end{array}\right.
$$

where $x \in \mathcal{R}^{n}$ is the state vector.

The main system $\Sigma_{1}$ and the subsystem $\Sigma_{2}$ is connected by connecting the output $z_{1}$ from $\Sigma_{1}$ to the input $w_{2}$ of $\Sigma_{2}$. The output $z_{2}$ of $\Sigma_{2}$ is connected to $w_{1}$ of $\Sigma_{1}$. This interconnection of $\Sigma_{1}$ and $\Sigma_{2}$ is shown in Fig. 1.

The interconnection of the two systems $\Sigma_{1}$ and $\Sigma_{2}$ shown in Fig. 1 gives directly the following condition on the diminsion of inputs and outputs:

$$
l_{1}=q_{2}, \quad l_{2}=q_{1}
$$

Rearranging the two systems $\Sigma_{1}$ and $\Sigma_{2}$, the interconnected system $\Sigma_{1} * \Sigma_{2}$ can be calculated and is given by:

$$
\left[\begin{array}{l}
e_{2} \\
e_{1} \\
y_{2} \\
y_{1}
\end{array}\right]=\mathcal{S}\left(\Sigma_{2}, \Sigma_{1}\right)\left[\begin{array}{l}
d_{2} \\
d_{1} \\
u_{2} \\
u_{1}
\end{array}\right]
$$




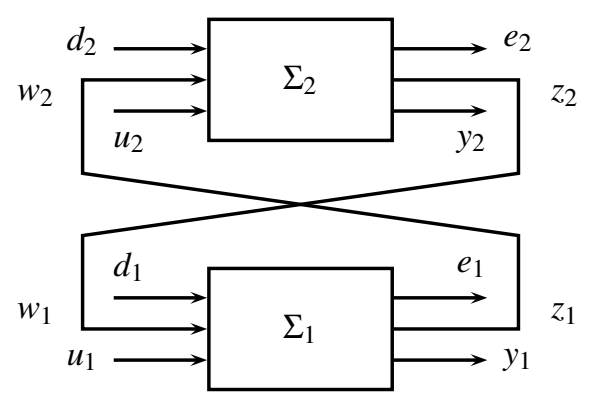

Fig. 1. Interconnection of system $\Sigma_{1}$ and $\Sigma_{2}$.

Let the main system $\Sigma_{1}$ be controllerd by a stabilizing controller, $\Sigma_{C, 1}$, i.e.

$$
\Sigma_{C, 1}:\left\{u_{1}=K_{1} y_{1}\right.
$$

Assume there exists a coprime factorization of the system $G_{y u, 1}$ and the stabilizing controller $K_{1}$ given by:

$$
\begin{array}{llr}
G_{y u, 1} & =N_{1} M_{1}^{-1}=\tilde{M}_{1}^{-1} \tilde{N}_{1}, \quad N_{1}, M_{1}, \tilde{N}_{1}, \tilde{M}_{1} \in \mathcal{R} \mathcal{H}_{\infty} \\
K_{1} & =U_{1} V_{1}^{-1}=\tilde{V}_{1}^{-1} \tilde{U}_{1}, \quad U_{1}, V_{1}, \tilde{U}_{1}, \tilde{V}_{1} \in \mathcal{R} \mathcal{H}_{\infty}
\end{array}
$$

The eight matrices will satisfy the double Bezout equation (see [7]).

Using the Bezout equation, all stabilizing controllers can be realized as an LFT (linear fractional transformation) in the YJBK transfer function $Q_{1} \in \mathcal{R} \mathcal{H}_{\infty}$ :

$$
K_{1}\left(Q_{1}\right)=\mathcal{F}_{l}\left(\left(\begin{array}{cc}
U_{1} V_{1}^{-1} & \tilde{V}_{1}^{-1} \\
V_{1}^{-1} & -V_{1}^{-1} N_{1}
\end{array}\right), Q_{1}\right)=\mathcal{F}_{l}\left(J_{K}, Q_{1}\right)
$$

This is shown in Fig. 2. All stabilizing controllers can also be realized through

$$
u_{1}=\tilde{V}^{-1}\left(\eta_{1}+\tilde{U} y_{1}\right)
$$

where:

$$
\eta_{1}=Q_{1} \varepsilon_{1}+\bar{\eta}_{1} \quad \varepsilon_{1}=\tilde{M} y_{1}-\tilde{N} u_{1}
$$

and $\bar{\eta}_{1}$ is an additional input vector.

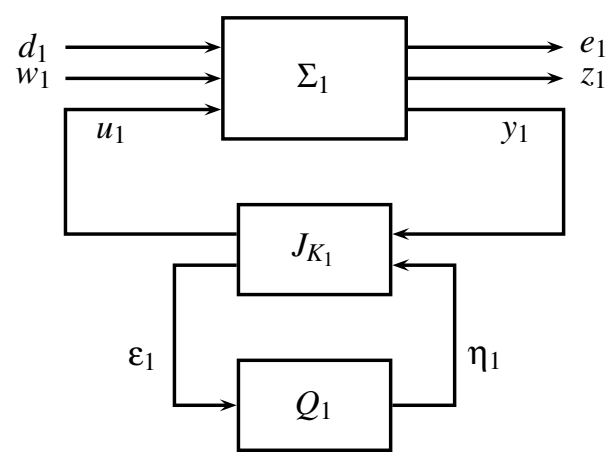

Fig. 2. A block diagram of $\Sigma_{1}$ controlled by $K_{1}\left(Q_{1}\right)$

\section{Closed-Loop StabiLity}

The stability of the closed-loop system is analyzed in the following when the sub-system $\Sigma_{2}$ is included in the loop. First, it is assumed that $\Sigma_{2}$ is known. Later, the stability analysis will be derived with an unknown $\Sigma_{2}$. It is assumed the $\Sigma_{1}$ is known in both cases.

Only the transfer function from $w_{2}$ to $z_{2}$ in $\Sigma_{2}$ is relevant in the following stability analysis. This gives the following simplified $\Sigma_{2}$ :

$$
\Sigma_{2}:\left\{z_{2}=G_{z w, 2} w_{2}\right.
$$

Only the case when a subsystem is included in the closedloop is investigated in the following. The analysis can also be used directly when a subsystem is removed from the closedloop system.

\section{A. $\Sigma_{2}$ is known}

When $\Sigma_{2}$ is known, it is simple to check the stability of the complete closed loop system. Closing the loop around the interconnected system given by (4) with the controller $K_{1}$, it is simple to calculate the closed-loop poles.

Instead of using the above standard method, it is possible to apply the dual YJBK parameterization in connection with a stability analysis. This can be done by calculating the dual YJBK transfer function $S$ for a certain change in the system. If $S$ is stable, the closed loop system is stable for the certain change in the system. Further, the dual YJBK transfer function will be applied later in connection with controller design.

The dual YJBK parameterization has been considered in details in [5], where also the connection between the dual YJBK transfer function and changes in the system has been described.

Using the above set-up, the dual YJBK transfer function is given by, [5]:

$$
S\left(G_{z w, 2}\right)=T_{21} G_{z w, 2}\left(I-T_{11} G_{z w, 2}\right)^{-1} T_{12}
$$

where

$$
\begin{aligned}
T_{11} & =G_{z w, 1}+G_{z u, 1} U_{1} \tilde{M}_{1} G_{y w, 1} \\
T_{12} & =G_{z u, 1} M_{1} \\
T_{21} & =\tilde{M}_{1} G_{y w, 1}
\end{aligned}
$$

$M_{1}, \tilde{M}_{1}$ and $U_{1}$ are the coprime matrices from the factorization of $G_{y u, 1}$ and $K_{1}$.

It is easy to see that $S\left(G_{z w, 2}\right)$ will be zero when $G_{z w, 2}$ is zero, i.e.

$$
S\left(G_{z w, 2}\right)=0, \text { for } G_{z w, 2}=0
$$

From the theory related to the dual YJBK parameterization the closed-loop system is stable if the nominal closed-loop system is stable and the dual YJBK transfer function is 
stable. The nominal closed-loop system is guaranteed to be stable by the design of $K_{1}$. This mean that the complete closed-loop system is stable if the dual YJBK transfer function given by (7) is stable. It is simple to calculate the close loop poles when the subsystem $\Sigma_{2}$ is known.

It is important to note that the above stability analysis is based on the information from the input $w_{2}$ and output $z_{2}$ on the subsystem $\Sigma_{2}$. Only the observable and controllable dynamic in $\Sigma_{2}$ is included in the analysis. This mean that parts of the subsystem that is not connected to the main system is unstable can be unstable without it is possible to see it through $S$.

\section{B. $\Sigma_{2}$ is unknown}

In the case when $\Sigma_{2}$ is unknown, it is not possible directly to calculate the closed-loop poles and the closed-loop stability can not be determined directly. Other methods can needs to be applied. Methods from robust control can be be applied. One method is to use the small gain theorem, [6], [9] for an investigation of the closed-loop stability. This methods require that $\Sigma_{2}$ is stable and an upper bound on gain is known.

Assume that the subsystem $\Sigma_{2}$ is open-loop stable. Let an upper bound on the gain of $\Sigma_{2}$ (or more correct of $G_{z w, 2}$ ) be given by:

$$
l_{G}(\omega) \geq \sigma_{\max }\left(G_{z w, 2}(j \omega)\right), \forall \omega
$$

$S\left(G_{z w, 2}\right)$ given by (7) is stable if $\left(I-T_{11} G_{z w, 2}\right)^{-1}$ is stable. $\left(I-T_{11} G_{z w, 2}\right)^{-1}$ is stable if

$$
\sigma_{\max }\left(T_{11}(j \omega)\right)<1 / l_{G}(\omega), \forall \omega
$$

(10) follows directly from using the small gain theorem on $S\left(G_{z w, 2}\right)$, [6], [9]. The small gain theorem has also been used in [3] in connection with stability anslysis and controller design for interconnected systems.

Another possibility is to estimate the subsystem $\Sigma_{2}$ through the dual YJBK transfer function $S\left(G_{z w, 2}\right)$ given by (7). $S\left(G_{z w, 2}\right)$ is an indirect measure of the subsystem $G_{z w, 2}$ connected to the system $\Sigma_{1}$. It is possible to calculate an upper bound on $S$ for when an upper bound on $G_{z w, 2}$ is known. This can be done by using the skew- $\mu$ method, [6].

$S\left(G_{z w, 2}\right)$ will not be used directly in connection with estimation of $G_{z w, 2}$. It is possible to modify the feedback controller $K_{1}$ by introduction a $Q_{1}$ as described in Section Fig. 2. The input vector $\eta_{1}$ is then given by:

$$
\eta_{1}=Q_{1} \varepsilon_{1}+\bar{\eta}_{1}
$$

The closed-loop transfer function from $\bar{\eta}_{1}$ to $\varepsilon_{1}$ is then given by:

$$
\begin{aligned}
S\left(Q_{1}, G_{z w, 2}\right) & =S\left(G_{z w, 2}\right)\left(I-Q_{1} S\left(G_{z w, 2}\right)\right)^{-1} \\
& =T_{21} G_{z w, 2}\left(I-\left(T_{11}+T_{12} Q_{1} T_{21}\right) G_{z w, 2}\right)^{-1} T_{12}
\end{aligned}
$$

Further, let's also include pre- and post-filters around $S\left(Q_{1}, \Delta\right)$ resulting in

$$
S_{W}\left(Q_{1}, G_{z w, 2}\right)=W_{O} S\left(Q_{1}, G_{z w, 2}\right) W_{I}
$$

This gives three transfer functions that can be designed with respect to get a simple and direct estimation of $G_{z w, 2}$. Based on (12) and (13), it is possible to apply standard estimation/identification methods. However, in the system set-up considered in this paper, it will in many cases be possible to simplify the above closed-loop transfer function such that it is possible to get a direct estimation of $G_{z w, 2}$.

Let the following conditions on the number of inputs and outputs for the two systems $\Sigma_{1}$ and $\Sigma_{2}$ be satisfied:

$$
\begin{aligned}
& l_{1}=q_{2} \leq m_{1} \\
& q_{1}=l_{2} \leq p_{1}
\end{aligned}
$$

The first condition guarantee that $G_{z u, 1}$ is right invertible. The second condition guarantee that $G_{y w, 1}$ is left invertible.

Let's consider the feedback part of $S_{W}\left(Q_{1}, G_{z w, 2}\right)$ given by:

$$
\left(T_{11}+T_{12} Q_{1} T_{21}\right) G_{z w, 2}
$$

The condition in (14) guarantee that a right and left inverse of $T_{12}$ and $T_{21}$ exists and given by $T_{12}^{\dagger}$ and $T_{21}^{\dagger}$, respectively.

Let $Q_{1}$ be given by

$$
Q_{1}=-T_{12}^{\dagger} T_{11} T_{21}^{\dagger}
$$

This gives directly that

$$
\left(T_{11}+T_{12} Q_{1} T_{21}\right) G_{z w, 2}=0
$$

Further, let the two filters $W_{I}$ and a $W_{O}$ be given by:

$$
W_{I}=T_{12}^{\dagger}, \quad W_{O}=T_{21}^{\dagger}
$$

Using the controller $Q_{1}$ given by (15) and the pre- and postfilter satisfying the conditions in (16) gives the following $S_{W}\left(Q, G_{z w, 2}\right)$ :

$$
S_{W}\left(Q_{1}, G_{z w, 2}\right)=G_{z w, 2}
$$

This decoupling of the internal feedback in $S_{W}\left(Q_{1}, G_{z w, 2}\right)$ by using $Q_{1}$ given by (15) is only allowed with a stable $Q_{1}$. A non-minimm phase zero in $T_{12}$ or $T_{21}$ must also be included in $T_{11}$ to give a stable $Q_{1}$. Necessary and sufficient conditions to give a stable $Q_{1}$ with respect to the original system can be derived but is not investigated further in this paper.

It is also required that the two weight functions $W_{I}$ and $W_{O}$ are stable. This will require that $T_{12}$ and $T_{21}$ does not include non-minimum phase zeros. If $T_{12}$ or $T_{21}$ include nonminimum phase zeros, it will be possible to make a factorization of the transfer functions, where they are factorized into a minimum phase part and an allpass part. The minimum phase part can be removed by selecting the weighting matrices as the inverse of these parts. The resulting transfer function 
from input to output will include the remaining all-pass factors. These can afterwards be removed manually.

A stabilizing controller $Q_{1}(s)$ given by (15) will give an exact decoupling as given by (17). Using (11), $G_{z w, 2}$ is then the transfer function between $\bar{\eta}_{1}$ and $\varepsilon_{1}$, i.e.

$$
\varepsilon_{1}=S_{W}\left(Q_{1}, G_{z w, 2}\right) \bar{\eta}_{1}=G_{z w, 2} \bar{\eta}_{1}
$$

Standard estimations methods can then be applied for the estimation of $G_{z w, 2}$.

\section{Controller Design}

Design of controllers for the interconnected system will be considered in this section. Beyond just designing a new controller for the complete interconnected system, there exist a number of alternatives to this. A complete new controller deign will have the drawback that it will require a complete new redesign again when the subsystem $\Sigma_{2}$ is removed again from the loop. It is there more relevant to consider controller architectures where the original controller $\Sigma_{C, 1}$ is preserved.

Two different design approaches will be considered in the following, where the nominal feedback controller is preserved. In the first approach, a controller is designed for a feedback around $\Sigma_{2}$. The controller must be designed such that the complete interconnected closed-loop system is stable. The other approach is to use the YJBK transfer function $Q_{1}$ for stabilizing the closed-loop system when $\Sigma_{2}$ is included.

Only the stability aspect in connection with the controller design will be considered in the following. The closed-loop performance can also be included, but will not be considered here in this paper.

\section{A. Local Controller Design for $\Sigma_{2}$.}

Considering a design of a local controller for $\Sigma_{2}$. Closing the loop from around $\Sigma_{2}$ by

$$
\Sigma_{C, 2}:\left\{u_{2}=K_{2} y_{2}\right.
$$

the closed loop system $\Sigma_{c l, 2}$ is then given by:

$$
\Sigma_{c l, 2}:\left\{\begin{array}{c}
e_{2}=T_{e d, 2}\left(K_{2}\right) d_{2}+T_{e w, 2}\left(K_{2}\right) w_{2} \\
z_{2}=T_{z d, 2}\left(K_{2}\right) d_{2}+T_{z w, 2}\left(K_{2}\right) w_{2}
\end{array}\right.
$$

where

$$
\begin{aligned}
& T_{e d, 2}\left(K_{2}\right)=G_{e d, 2}+G_{e u, 2} K_{2}\left(I-G_{y u, 2} K\right)^{-1} G_{y d, 2} \\
& T_{e w, 2}\left(K_{2}\right)=G_{e w, 2}+G_{e u, 2} K_{2}\left(I-G_{y u, 2} K\right)^{-1} G_{y w, 2} \\
& T_{z d, 2}\left(K_{2}\right)=G_{z d, 2}+G_{z u, 2} K_{2}\left(I-G_{y u, 2} K\right)^{-1} G_{y d, 2} \\
& T_{z w, 2}\left(K_{2}\right)=G_{z w, 2}+G_{z u, 2} K_{2}\left(I-G_{y u, 2} K\right)^{-1} G_{y w, 2}
\end{aligned}
$$

The local feedback controller $K_{2}$ must be designed such that the complete closed-loop system is stable. This require that $K_{2}$ must be designed such that $S\left(K_{2}\right)$ given by

$$
S\left(K_{2}\right)=T_{21} T_{z w, 2}\left(K_{2}\right)\left(I-T_{11} T_{z w, 2}\left(K_{2}\right)\right)^{-1} T_{12}
$$

must be stable.

$S\left(K_{2}\right)$ can be written in the following form:

$$
\begin{aligned}
S\left(K_{2}\right) & =\mathcal{F}_{u}\left(\left(\begin{array}{cc}
T_{11} & T_{12} \\
T_{21} & 0
\end{array}\right), \mathcal{F}_{u}\left(\left(\begin{array}{ll}
G_{y u, 2} & G_{y w, 2} \\
G_{z u, 2} & G_{z w, 2}
\end{array}\right), K_{2}\right)\right) \\
& =\mathcal{F}_{u}\left(\left(\begin{array}{cc}
T_{S, 11} & T_{S, 12} \\
T_{S, 21} & T_{S, 22}
\end{array}\right), K_{2}\right)
\end{aligned}
$$

where

$$
\begin{aligned}
T_{S, 11} & =G_{y u, 2}+G_{y w, 2} T_{11}\left(I-G_{z w, 2} T_{11}\right)^{-1} G_{z u, 2} \\
T_{S, 12} & =G_{y w, 2}\left(I-T_{11} G_{z w, 2}\right)^{-1} T_{12} \\
T_{S, 21} & =T_{21}\left(I-G_{z w, 2} T_{11}\right)^{-1} G_{z u, 2} \\
T_{S, 22} & =T_{21} G_{z w, 2}\left(I-T_{11} G_{z w, 2}\right)^{-1} T_{12}
\end{aligned}
$$

Now, standard methods can be applied to design a feedback controller $K_{2}$ that will stabilize the complete closed-loop system. Necessary and sufficient conditions to guarantee that this can be obtained has not been investigated at this time.

\section{B. Redesign of $\Sigma_{C, 1}$ by $Q_{1}$.}

As an alternative to design a local controller directly for $\Sigma_{2}$, it is possible to redesign $\Sigma_{C, 1}$ by using the YJBK transfer function $Q_{1}$. This will again leave the original feedback controller for $\Sigma_{1}$ unchanged.

The closed-loop system is stable if the nominal closed-loop is stable and the closed-loop given by $S\left(\left(Q_{1}, G_{z w, 2}\right)\right.$ given in (12) is stable. $S\left(\left(Q_{1}, G_{z w, 2}\right)\right.$ can also be written in an LFT form given by

$$
S\left(\left(Q_{1}, G_{z w, 2}\right)=\mathcal{F}_{l}\left(\left(\begin{array}{cc}
S\left(G_{z w, 2}\right) & S\left(G_{z w, 2}\right) \\
S\left(G_{z w, 2}\right) & S\left(G_{z w, 2}\right)
\end{array}\right), Q_{1}\right)\right.
$$

Again, standard methods can be applied for the deisgn of $Q_{1}$ that will stabilize the closed-loop system.

\section{EXAMPLE}

The example is a mass spring system. The main system $\Sigma_{1}$ consist of a two mass spring systems and the sub system $\Sigma_{2}$ is a single mass spring system. The two mass spring system described in [9]. This system has the following state space description:

$$
\begin{aligned}
A_{1} & =\left(\begin{array}{cccc}
0 & 0 & 1 & 0 \\
0 & 0 & 0 & 1 \\
-1 & 1 & -0.2 & 0.2 \\
0.5 & -2.5 & 0.1 & -0.15
\end{array}\right) \\
\left(\begin{array}{cc}
B_{d, 1} & B_{u, 1}
\end{array}\right)= & \left(\begin{array}{cc}
0 & 0 \\
0 & 0 \\
0 & 1 \\
0.5 & 0
\end{array}\right) \\
C_{e, 1}=C_{y, 1} & =\left(\begin{array}{cccc}
1 & 0 & 0 & 0 \\
0 & 1 & 0 & 0
\end{array}\right)
\end{aligned}
$$


An observer based feedback controller is applied to the system with the following state feedback gain $F$ and observer gain $L$ :

$$
\begin{aligned}
& F=\left(\begin{array}{llll}
-0.6886 & -1.1124 & -0.6288 & 0.0837
\end{array}\right) \\
& L=\left(\begin{array}{cc}
-8.3733 & 16.2211 \\
-0.3220 & -5.2795 \\
2.1388 & -70.0381 \\
53.0028 & -195.6965
\end{array}\right)
\end{aligned}
$$

The observer based feedback controller is based on an $H_{\infty}$ feedback controller designed in [9]. The controller is transformed into an observer based feedback controller by using the method described in [1].

A mass spring system $\Sigma_{2}$ is connected to the main system $\Sigma_{1}$ through the output $z_{1}$ and the input $w_{1}$. The associated output and input matrices are given by:

$$
C_{z, 1}=\left(\begin{array}{llll}
1 & 0 & 0 & 0
\end{array}\right), B_{w, 1}=\left(\begin{array}{l}
0 \\
0 \\
1 \\
0
\end{array}\right)
$$

Further, the transfer function $G_{z w, 2}$ is given by

$$
G_{z w, 2}=\frac{\left(k_{3}+b_{3} s\right)^{2}}{m_{3} s^{2}+b_{3} s+k_{3}}
$$

with $m_{3}=0.5, K_{3}=1$ and $b_{3}=0.1$.

Using an observer based feedback controller, $T_{11}, T_{12}$ and $T_{21}$ are given by:

$$
\begin{aligned}
T_{11}= & C_{z, 1}\left(s I-A-B_{u, 1} F\right)^{-1} L C_{y, 1}\left(s I-A-L C_{y, 1}\right)^{-1} B_{w, 1} \\
& +C_{z, 1}\left(s I-A-L C_{y, 1}\right)^{-1} B_{w, 1} \\
T_{12}= & C_{z, 1}\left(s I-A-B_{u, 1} F\right)^{-1} B_{u, 1} \\
T_{21}= & C_{y, 1}\left(s I-A-L C_{y, 1}\right)^{-1} B_{w, 1}
\end{aligned}
$$

In the following, let's consider the case where the YJBK transfer function $Q_{1}$ is designed such that $S\left(G_{z w, 2}\right)=G_{z w, 2}$. This is possible in this case, because neither $T_{12}$, nor $T_{21}$ include RHP zeros. However, both $T_{12}$ and $T_{21}$ are strictly proper, so the inverses of the two transfer functions does not exist. Instead, an approximation at low frequencies is used. This is obtained by including a small direct term in $T_{12}$ and $T_{21}$. This will affect the result at high frequencies.

In the case $Q_{1}$ is given by

$$
Q_{1}=\left(\begin{array}{ll}
Q_{11} & Q_{12}
\end{array}\right)
$$

In this case it is possible to let one of the transfer functions in $Q_{1}$ to be equal to zero and still get a decoupling. Here we let $Q_{12}=0$. The nominal feedback controller is modified by using $Q_{11}$ such that the dual YJBK transfer function gets equal to $G_{z w, 2}$. This is obtained by using $Q_{11}, W_{I}$ and $W_{O}$ given by (15) and (16).

Some simulations are shown in the following. A step input is injected into the system through the input $\eta_{1}$. The output responses for mass 1 and mass 2 in $\Sigma_{1}$ is shown in Fig. 3 . In Fig. 4, the outputs are shown when the sub-system $\Sigma_{2}$ is included. It can be seen from the output responses in Fig. 3 and Fig. 4 that the introduction of the sub-system $\Sigma_{2}$ has a major effect on the output responses.

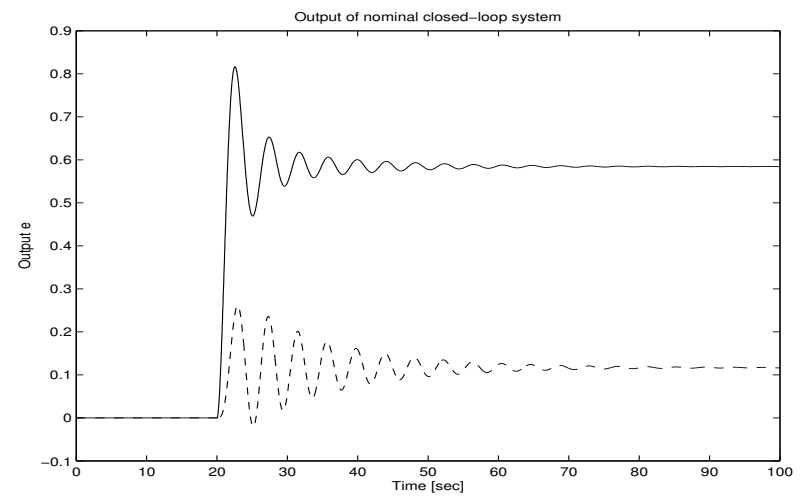

Fig. 3. The positions of mass 1 and 2 of $\Sigma_{1}$ for a step input on $\eta$. The solid line is the output for mass no. 1 and the dashed line is the output for mass no. 2.

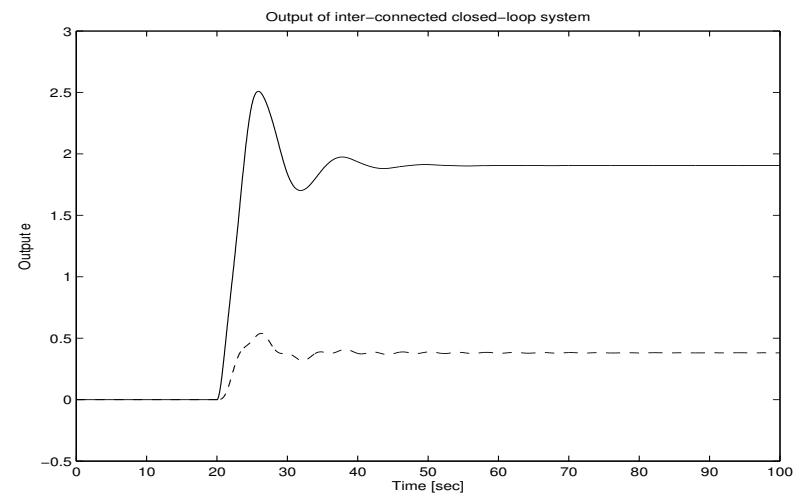

Fig. 4. The position of mass 1 and 2 of $\Sigma_{1}$ for a step input on $\eta$ when the subsystem $\Sigma_{2}$ is connected. The solid line is the output for mass no. 1 and the dashed line is the output for mass no. 2 .

This can be investigated further by considering the transfer function from $\eta_{1}$ to $\varepsilon_{1}$, i.e. the dual YJBK transfer function. The output response of $\varepsilon_{1}$ for a step on $\eta_{1}$ when $\Sigma_{2}$ is included is shown in Fig. 5. The response shows that $S\left(G_{z w, 2}\right)$ is stable.

Including $Q_{11}, W_{I}$ and $W_{O}$ gives the step response shown in Fig. 6. The step response is compared with a step response directly from $G_{z w, 2}$. There is only a minor difference between the two step responses. This show that $S_{W}\left(Q_{1}, G_{z w, 2}\right) \approx G_{z w, 2}$. This can also be seen from the Bode plot shown in Fig. 6 and Fig. 7. Here, the amplitudes and the phases of $S_{W}\left(Q_{1}, G_{z w, 2}\right)$ and $G_{z w, 2}$ are shown. $S_{W}\left(Q_{1}, G_{z w, 2}\right)$ is calculated out from a Simulink model by using the Matlab function "linmod" followed by a model reduction. Due to the approximation of the inverses of $T_{12}$ and $T_{21}$, the two set of curves are almost identical at low frequencies. For $\omega \geq 10 \mathrm{rad} / \mathrm{sec}$, there is a major difference between the curves for $S_{W}\left(Q_{1}, G_{z w, 2}\right)$ and $G_{z w, 2}$. 


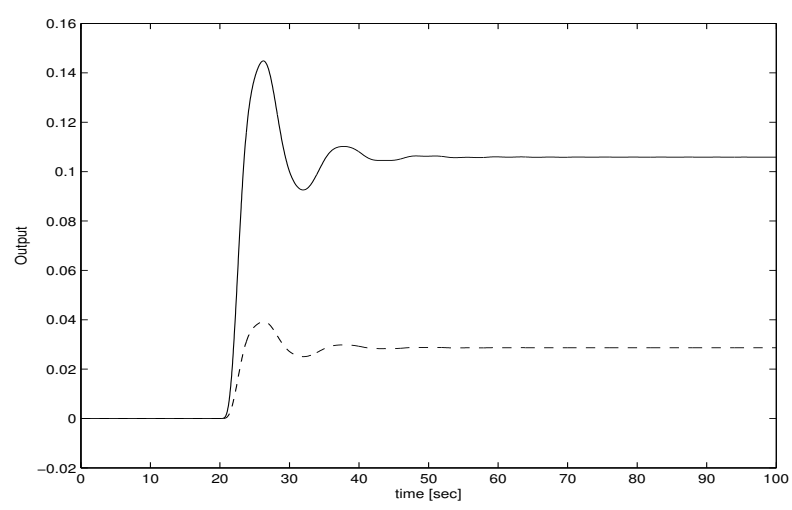

Fig. 5. The output response $\varepsilon_{1}$ for a step input on $\eta_{1}$ when $\Sigma_{2}$ is included.

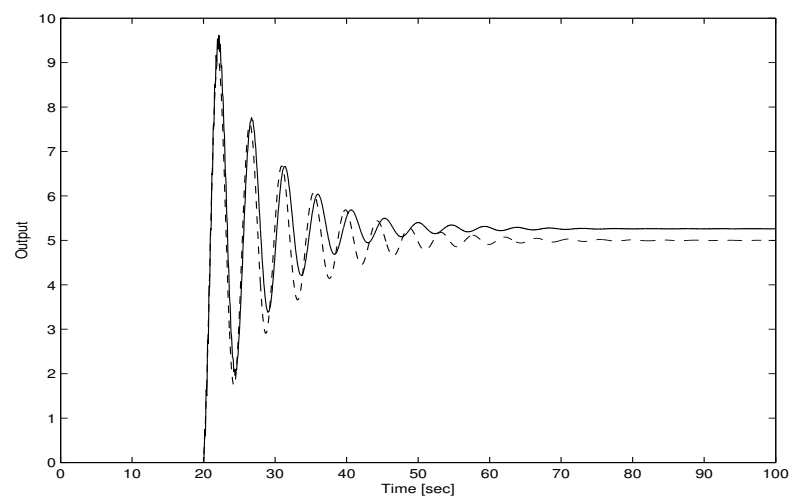

Fig. 6. Step reponse of $S_{W}\left(Q_{1}, G_{z w, 2}\right)$ (solid line) and of the subsystem $\Sigma_{2}$ (dashed line).

\section{Discussion}

Some new analysis and controller design results for closedloop interconnected systems has been presented here. The key element in both the analysis and the design is a application of the YJBK and the dual YJBK parameterization. The closed-loop stability analysis is based directly on the dual YJBK transfer function. Analysis results are given in both cases where the sub-system is known and in the case where it is unknown. Further, it is shown that it is possible to design the YJBK transfer function, pre- and post-filters such that a connected sub-system can be estimated directly without having a direct access to the sub-system.

The last, design of controllers has been investigated. Two different controller concepts have been described. In the first case, a local controller is designed for the sub-system. In the other case, the existing controller for the main system is modified by using the YJBK transfer function. In both cases, the nominal controller is not changed by the new controllers.

Only the closed-loop stability has been considered in this paper. The next step is to include the performance of the interconnected in both the analysis and the controller design.

Another area for further research is a more detailed investigation of the conditions for obtaining an exact or an almost

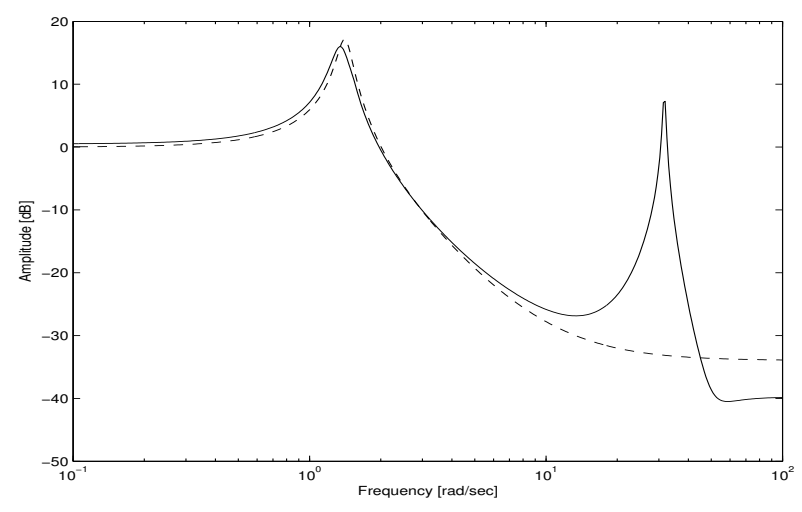

Fig. 7. The amplitude of $S_{W}\left(Q_{1}, G_{z w, 2}\right)$ and of the sub-system $\Sigma_{2}$ (dashed line).

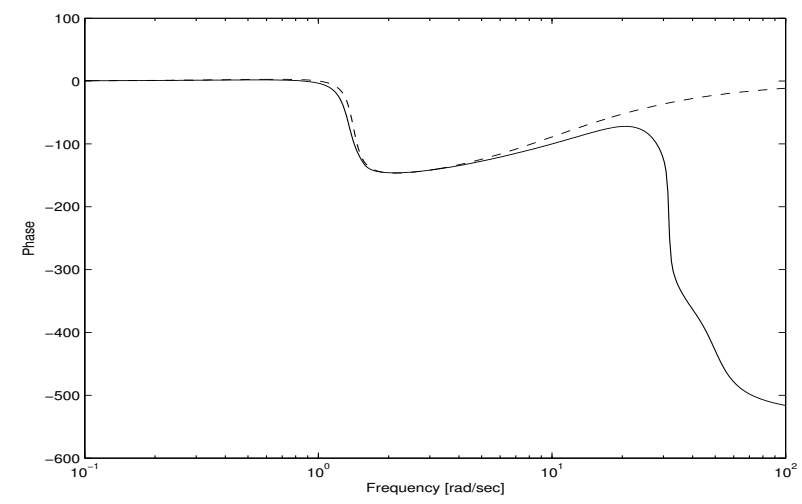

Fig. 8. The phase of $S_{W}\left(Q_{1}, G_{z w, 2}\right)$ and of the sub-system $\Sigma_{2}$ (dashed line).

exact decoupling in connection with the estimation of the connected sub-system.

\section{REFERENCES}

[1] D. Alazard and P. Apkarian. Exact observer-based structures for arbitrary compensators. International Journal of Robust and Nonlinear Control, 9:101-118, 1999.

[2] R. D'Andrea and G.E. Dullerud. Distributed control design for spatially interconnected systems. IEEE Transactions on Automatic Control, 48(9):1478-1495, 2003.

[3] Z. Duan, L. Huang, L. Wang, and J. Wang. Some applications of small gain theorem to interconnected systems. Systems \& Control Letters, 52(3-4):263-273, 2004.

[4] L. Guzzella. Automative systems - an automatic control bonanza. In Proceedings of the American Control Conference, Seattle, WA, USA, 2008. Plenary lecture.

[5] H.H. Niemann. Dual Youla parameterization. IEE Proceedings Control Theory and Applications, 150(5):493-497, 2003.

[6] S. Skogestad and I. Postlethwaite. Multivariable feedback control: Analysis and Design. Wiley, 2005.

[7] T.T. Tay, I.M.Y. Mareels, and J.B. Moore. High performance control. Birkhäuser, 1997.

[8] J.C. Willems. The behavioral approach to open an interconnected systems. IEEE Control System Magazine, 27(6):46-99, 2007.

[9] K. Zhou. Essentials of Robust Control. Prentice Hall, 1998.

[10] Y. Zhu and P.R. Pagilla. Decentralized output feedback control of a class of large-scale interconnected systems. IMA Journal of Mathematical Control and Information, 24(1):57-69, 2007. 